\title{
Comment on " $\eta_{c}$ production in photon-induced interactions at the LHC"
}

\author{
Spencer R. Klein * \\ Nuclear Science Division, Lawrence Berkeley National Laboratory, \\ Berkeley, California 94720, USA
}

(Received 17 August 2018; published 14 December 2018)

\begin{abstract}
In " $\eta_{c}$ production in photon-induced interactions at the LHC," [Phys. Rev. D 97, 094009 (2018)] Goncalves and Moreira discuss inclusive and exclusive $\eta_{c}$ production at $p p$ and $p A$ collisions at LHC energies. The exclusive channels are via two-photon and photon-Odderon interactions. This comment points out that there is a large additional source of almost-exclusive $\eta_{c}$ in ultraperipheral collisions: from the radiative decay of $J / \psi$ that are produced in photon-nucleon interactions. Although the $J / \psi \rightarrow \gamma \eta_{c}$ branching ratio is small, the $J / \psi$ production cross-section is large enough that it dominates over the exclusive channels considered in [1], and is comparable to the nonexclusive production. In $J / \psi \rightarrow \gamma \eta_{c}$, the photon is very soft and therefore easy to miss, and the $\eta_{c}$ will have very similar kinematics to the $J / \psi$.
\end{abstract}

DOI: 10.1103/PhysRevD.98.118501

Two-photon production of the $\eta_{c}$ has long been a target for ultraperipheral collisions at heavy ion colliders [2-4], and early RHIC [5] and ALICE [6] studies considered its production in the two-photon channel. Production via double-Pomeron (also called central exclusive production) has also been considered [7]. These calculations found rather small cross-sections, leading to a decline in interest.

Goncalves and Moreira [1] consider $p p$ and $p A$ collisions, via both two-photon and photonuclear channels, finding that inclusive $\gamma p$ interactions dominate, with cross sections of $3.492 \mathrm{nb}$ in $p p$ collisions at $\sqrt{s}=13 \mathrm{TeV}$, and $3.194 \mu \mathrm{b}$ in $p P b$ collisions at $\sqrt{s}=8.1 \mathrm{TeV}$. The total inclusive rates (two-photon + photon-Odderon [8]) are much smaller, $0.059 \mathrm{nb}$ and $0.501 \mathrm{nb}$ respectively for $p p$ collisions, and $0.182 \mu \mathrm{b}$ and $0.351 \mu \mathrm{b}$ for $p P b$ collisions. The existence of the Odderon is rather speculative, so it is important to consider whether there are any exclusive production channels with cross-sections larger than the $\gamma \gamma$ channel, to avoid possible false claims for the existence of the Odderon.

As Sec. 4.4 of Ref. [9] pointed out, the rate for coherent $J / \psi$ photoproduction, followed by the decay $J \psi \rightarrow \gamma \eta_{c}$ is larger than that for two-photon production of the $\eta_{c}$. Because the emitted photon is so soft (energy of

*srklein@lbl.gov

Published by the American Physical Society under the terms of the Creative Commons Attribution 4.0 International license. Further distribution of this work must maintain attribution to the author(s) and the published article's title, journal citation, and DOI. Funded by SCOAP.
$111 \mathrm{MeV}$ in the $J / \psi$ rest frame), it is likely to be missed in any LHC detector, and the $\eta_{c}$ will have a similar rapidity and transverse momentum as the $J / \psi$ parent, so the final state will look like exclusive photoproduction.

Here, I use STARlight [10] to make a similar calculation for the $p p$ and $p A$ collisions discussed in Ref. [1]. STARlight uses a fairly standard model for photoproduction [11], using the Weizsacker-Williams photon flux and requiring that the two nuclei do not interact hadronically. For $p p$ collisions, STARlight uses a photon flux [12] similar to that used by Goncalves and Moreira. STARlight does not include nuclear shadowing, but, since the bulk of the production in $p A$ collisions comes when the proton is a target, shadowing should not be important here, and STARlight has been shown to accurately predict $J / \psi$ photoproduction at the LHC, in both $p P b$ [13] and $p p$ collisions [14].

Table I gives the coherent photoproduction crosssections for $J / \psi$ and $\eta_{c}$ production cross sections for these $p p$ and $p P b$ collisions, based on the branching ratio $\operatorname{Br}\left(\eta_{c} \rightarrow \gamma \eta_{c}\right)=0.17$ [15].

TABLE I. The cross-sections for $J / \psi$ photoproduction in $p p$ and $p P b$ collisions at the LHC (with separate lines for photon emission by the proton and the lead nucleus), along with the production cross section for the $\eta_{c}$ in all three modes.

\begin{tabular}{lcc}
\hline \hline & $\sigma(J / \psi)$ & $\sigma\left(J / \psi \rightarrow \eta_{c} \gamma\right)$ \\
\hline$p p$ & $79 \mathrm{nb}$ & $1.34 \mathrm{nb}$ \\
$p P b$ (proton-shine) & $3.21 \mu \mathrm{b}$ & $54 \mathrm{nb}$ \\
$p P b$ (lead-shine) & $57.5 \mu \mathrm{b}$ & $1.0 \mu \mathrm{b}$ \\
\hline \hline
\end{tabular}



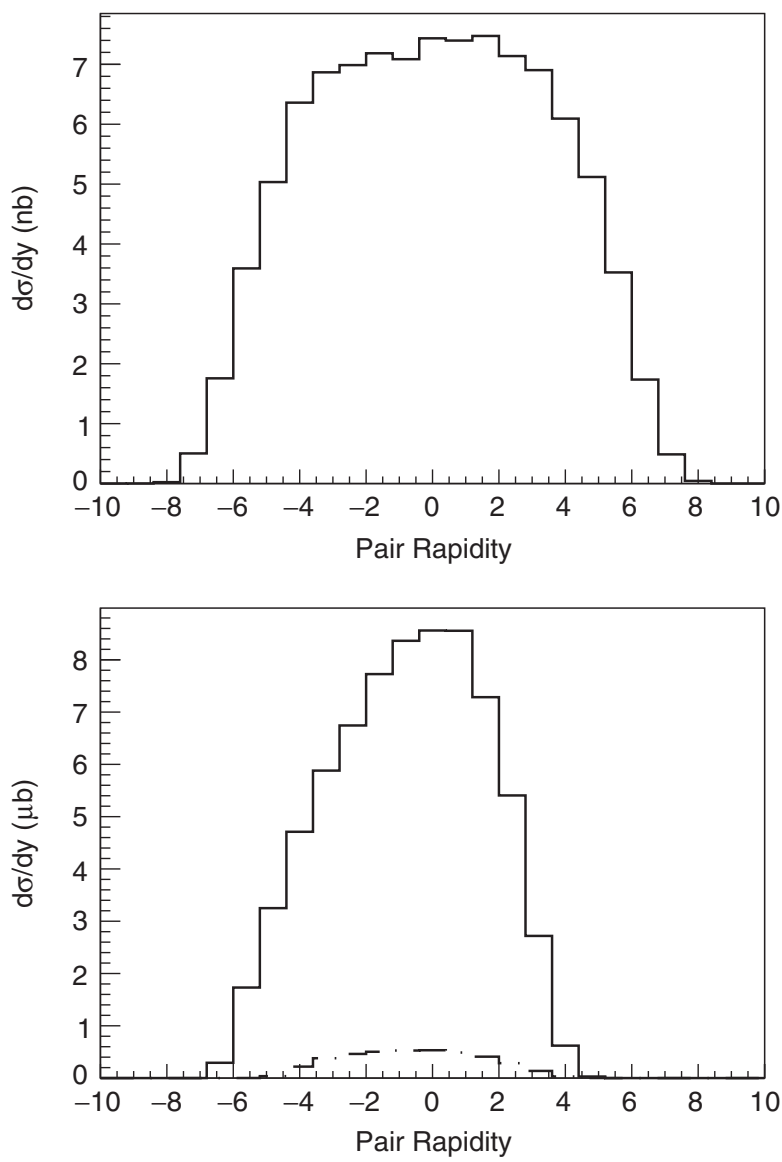

FIG. 1. The rapidity distribution $d \sigma / d y$ for $J / \psi$ photoproduction at the LHC for (top) $p p$ collisions at $\sqrt{s}=13 \mathrm{TeV}$ and (bottom) $p P b$ collisions at $\sqrt{s_{N N}}=8.1 \mathrm{TeV}$. In the bottom plot, the solid black curve is "lead-shine," and the dashed dark blue curve is "proton-shine".
For both $p p$ and $p P b$ collisions, the $\eta_{c}$ rate is higher than the exclusive channels considered in Goncalves and Moreira. The cross sections are also higher than the nonexclusive diffractive production channel (which also leaves both beam particles intact).

Figure 1 shows the rapidity distribution for $J / \psi$ produced in $p p$ and $p A$ collisions; the latter is separated out by photon emitter. The bulk of the $p P b$ production occurs when photons are emitted by the lead nucleus. Both overall distributions are quite similar to the $d \sigma / d y$ for diffractive photoproduction in Fig. 4 of Ref. [1].

One way to differentiate between $\gamma \gamma$ and photonPomeron (or photon-Odderon) production is by examining the transverse momentum distribution, $p_{T}$, of the $\eta_{c}$. Twophoton production has a considerably smaller $p_{T}$ scale than the photoproduction channels [16], particularly when a proton is the photoproduction target. Photon-Pomeron and photon-Odderon processes should have a similar $p_{T}$ spectra, so be indistinguishable.

These cross sections are considerably larger than any of the exclusive $\eta_{c}$ cross-sections discussed in Ref. [1], and are the most likely source for apparent exclusive $\eta_{c}$. Similar conclusions will apply for collisions of other beam particles [8]. It is important that $J / \psi$ photoproduction be considered; otherwise, any observation of exclusive $\eta_{c}$ beyond that expected from two-photon physics might be mistaken for a more exotic process.

This work was funded by the U.S. DOE under Contract No. DE-AC02-05-CH11231.
[1] V. P. Gonalves and B. D. Moreira, Phys. Rev. D 97, 094009 (2018).

[2] N. Baron and G. Baur, Phys. Rev. C 49, 1127 (1994); The results here are consistent with other results if one wrongly takes $\hbar c=1$, in units of GeV-fm; Gerhard Baur (private communication).

[3] M. Vidovic, M. Greiner, and G. Soff, J. Phys. G 21, 545 (1995).

[4] A. J. Schramm and D. H. Reeves, Phys. Rev. D 53, 4092 (1996).

[5] J. Nystrand et al., arXiv:nucl-ex/9811007.

[6] S. Sadkovsky, Report No. ALICE/93-07, 1993.

[7] A. J. Schramm and D. H. Reeves, Phys. Rev. D 55, 7312 (1997).

[8] V. P. Goncalves, Nucl. Phys. A902, 32 (2013).
[9] C. A. Bertulani, S. R. Klein, and J. Nystrand, Annu. Rev. Nucl. Part. Sci. 55, 271 (2005).

[10] S. R. Klein, J. Nystrand, J. Seger, Y. Gorbunov, and J. Butterworth, Comput. Phys. Commun. 212, 258 (2017).

[11] S. Klein and J. Nystrand, Phys. Rev. C 60, 014903 (1999).

[12] S. R. Klein and J. Nystrand, Phys. Rev. Lett. 92, 142003 (2004).

[13] J. Adam (ALICE Collaboration), Proc. Sci., DIS2016 (2016) 187.

[14] LHCb Collaboration, Report No. LHCb-CONF-2016-007, http://inspirehep.net/record/1482815?ln=en.

[15] C. Patrignani et al. (Particle Data Group), Chin. Phys. C 40, 100001 (2016).

[16] A. J. Baltz, Y. Gorbunov, S. R. Klein, and J. Nystrand, Phys. Rev. C 80, 044902 (2009). 